\title{
Errors in health care management: what do they cost?
}

\author{
K D Rigby, J C B Litt
}

\begin{abstract}
Background-Iatrogenic injuries are relatively common and a potentially avoidable source of morbidity. The economic evaluation of this area has been limited by the lack of good quality national data to provide an estimate of incidence, associated disability, and preventability of iatrogenic injuries. Two recent surveys, the Quality in Australian Health Care Study (QAHCS) and the Utah Colorado Study (UTCOS), have now made this feasible.

Aims-To determine the direct costs associated with iatrogenic injuries occurring in a hospital setting.

Methods-The QAHCS was used as a representative national source of information on the incidence, disability, and preventability of iatrogenic injuries. Costs were calculated using information from Australian disease related groups (AN-DRGs) relative to the injury categories.

Results-The cost of just 12 preventable iatrogenic injuries is significant $(0.25$ million US dollars) and accounts for $2-3 \%$ of the annual budget of a typical Australian community based hospital of 120 beds. Costing data provide additional useful information for policy and decision makers.

Conclusion-Costing iatrogenic injuries is an important component of the impact of these events. An ongoing national database of iatrogenic injuries is necessary to assist in identifying the incidence of these injuries, monitoring trends, and providing data for cost estimates and economic evaluations.

(Quality in Health Care 2000;9:216-221)
\end{abstract}

Formerly Australian Patient Safety Foundation, GPO Box 511, Stirling, South Australia, Australia K D Rigby, health economist and research analyst

Department of General Practice, Flinders University, Noarlunga Hospital, Noarlunga, South Australia, Australia J C B Litt, general practitioner and senior lecturer

Correspondence to: Dr J C B Litt

jlitt@flinders.edu.au

Accepted 3 October 2000
Keywords: iatrogenic injuries; costs

Iatrogenic injuries are a potentially avoidable source of morbidity and mortality ${ }^{1}$ which affect a significant number of patients both in institutional care and in the community. ${ }^{2}$ A proportion of these patients will suffer permanent disability and some will die. ${ }^{2}$ Iatrogenic injuries can be defined as "unintended or unnecessary harm or suffering arising from any aspect of health care management". ${ }^{3}$ They arise from a therapeutic intervention and result either from systems or from human error, or a combination of the two. They can be distinguished from adverse or side effects, which are unavoidable or unpredictable events that occur during the appropriate application of best practice. Specifically, iatrogenic injuries are events that arise as a result of incomplete or inappropriate diag-
Key messages

- The economic burden of iatrogenic injuries is significant.

- There is still debate on what constitutes an injury.

- Economic evaluations of health care technologies should include the consequences and costs of iatrogenic injuries.

What this paper adds to the area Iatrogenic injuries are avoidable and negative consequences of usual clinical practice, arising from health care management; however, the nature and extent of the morbidity (and associated cost) occurring from iatrogenic injury has been infrequently documented. Economic evaluation of health care technologies that fail to take into account the consequences and costs of iatrogenic injuries may result in inefficient adoption of health care interventions. While there is a need to replicate surveys such as the QAHCS and UTCOS in other settings, further debate on what constitutes an iatrogenic injury is required.

nosis or therapeutic interventions. The untoward event is potentially preventable.

Estimating the incidence and calculating the costs of iatrogenic injuries (henceforth referred to as injuries or injury) from the perspective of the health care system is now possible since the availability of the Quality in Australian Health Care Study (QAHCS) ${ }^{1}$ and Utah Colorado Study (UTCOS) ${ }^{24}$ data sets. Initially, the QAHCS revealed higher levels of injuries than its American counterpart, but subsequent analysis has shown that the Australian study had taken a broad quality of care approach rather than focusing on negligence and compensation. ${ }^{5}$ Although this type of data is not yet available elsewhere, findings from the QAHCS and UTCOS should be applicable to other health care systems.

"Hidden" costs of iatrogenic injury and why this information is important to health economic evaluations

Economic evaluations compare the costs and benefits (or outcomes) of alternative ways of providing health care programmes and interventions. $^{6}$ Ideally, all alternatives are compared with usual clinical care or the next best alternative. ${ }^{6}$ Estimation of the "costs" of side or adverse effects is measured directly as part of an experimental or observational study or it is modelled probabilistically from the literature or expert opinion. Side or adverse effects 
are defined as "a result of drug or other therapy in addition to or in extension of the desired therapeutic effect". ${ }^{7}$ Generally, the use of these terms is more narrowly applied and does not include iatrogenic injuries that occur in usual clinical care.

The measurement of side or adverse effects in clinical trials is conducted under heavily scrutinised circumstances where the application of programmes or drugs is closely monitored and regulated to maximise internal validity. Such surveillance is likely to minimise the incidence of iatrogenic injuries. In usual clinical practice the level of surveillance for adverse outcomes is less rigorous and iatrogenic injuries may pass unnoticed.

To date, iatrogenic injuries have not been considered as an independent cost category in economic evaluations and differ from complications or side/adverse effects. There are a number of reasons for considering the impact of iatrogenic injures: (1) iatrogenic injuries, unlike side or adverse effects, are not a normal or expected consequence of a therapeutic or clinical intervention; (2) many of these injuries are avoidable or preventable and therefore impose an unnecessary burden on society; and (3) the relative cost effectiveness of competing alternatives in economic evaluations may be underestimated or overestimated without inclusion of the costs and consequences of iatrogenic injuries.

The lack of national data on the incidence of injuries has limited investigators to estimating the costs of injuries associated with specific therapeutic interventions, ${ }^{8-10}$ patient subsets, ${ }^{11}{ }^{12}$ or regions ${ }^{13}$ rather than to a health care system generally. The application of health economics to injuries involves two components-firstly, a descriptive component which determines the burden of these injuries on health care providers, funders, patients, and society in general, and secondly, an analytical component which weighs up the costs and benefits (or outcomes) of preventing, avoiding, or minimising the impact of injuries in part or in their entirety. This article focuses on the former component and is the first attempt to determine the direct costs of injuries arising within hospitals and in those admitted from the community with an injury.

\section{Methods}

The QAHCS was a retrospective random sample review of 14179 medical records from 28 Australian acute care hospitals in $1992 .^{1}$ Patients admitted with an injury from the community (including nursing homes) and inpatients were included. Two and, in some cases, three medical reviewers in the QAHCS survey independently assigned a "score" of disability and preventability for every injury identified in the survey based upon the criteria outlined in boxes 1 and 2 .

To improve the generalisability of the results of this analysis the aggregated preventability and disability scores for each injury category were based only upon those injuries with a score of between 5 and 6 and those with disability scores of between 4 and 8 . This
An injury can either increase the period of hospitalisation or cause a disability at the time of discharge, or both. Impairment ranging from temporary to permanent and any disability lasting more than a year should be considered a permanent impairment.

Based on the evidence in the medical notes, how would you judge the degree of disability attributable to the adverse event? $1=$ Minimal impairment and/or recovery in one month

$2=$ Moderate impairment, recovery in 1-6 months

3 = Moderate impairment, recovery in 6-12 months

$4=$ Permanent impairment, disability $1-50 \%$

$5=$ Permanent impairment, disability $>50 \%$

$6=$ Permanent nursing

$7=$ Institutional care

8 = Death

9 = Cannot reasonable judge

Box 1 Disability criteria for reviewers (QAHCS)

For an injury to be judged preventable it could have been averted with different management or treatment (failure to follow accepted practice and could be system, process, or human error).

Rate on a 6 point scale your confidence in the evidence of preventability.

$1=$ Virtually no evidence for preventability

$2=$ Slight to modest evidence for preventability

3 = Preventability not quite likely; less than $50 / 50$ but close call

4 = Preventability more likely than not; more than $50 / 50$ but close call

$5=$ Strong evidence of preventability

6 = Virtually certain evidence of preventability

Box 2 Preventability criteria for reviewers (QAHCS)

ensured that the injury qualified as having "good evidence" of preventability and at least some degree of permanent impairment.

Twelve injuries were randomly selected from the top 25 identified from the QAHCS. The mean of the reviewers' scores for disability and preventability was calculated, together with the estimated incidence data (along with 95\% confidence intervals). The data were extrapolated to arrive at the expected number of injuries, permanent disability, and preventability per 10000 hospital discharges.

An average direct acute care cost per category using Australian disease related groups (AN-DRG version 3) data was calculated per 10000 discharges. ${ }^{14}$ Principal diagnosis cost weights were used except in the case of warfarin related injuries, inadequate manipulation of fractures, and unnecessary operations. The heterogeneous nature of these latter categories required more refined costings using secondary diagnoses. The costing 

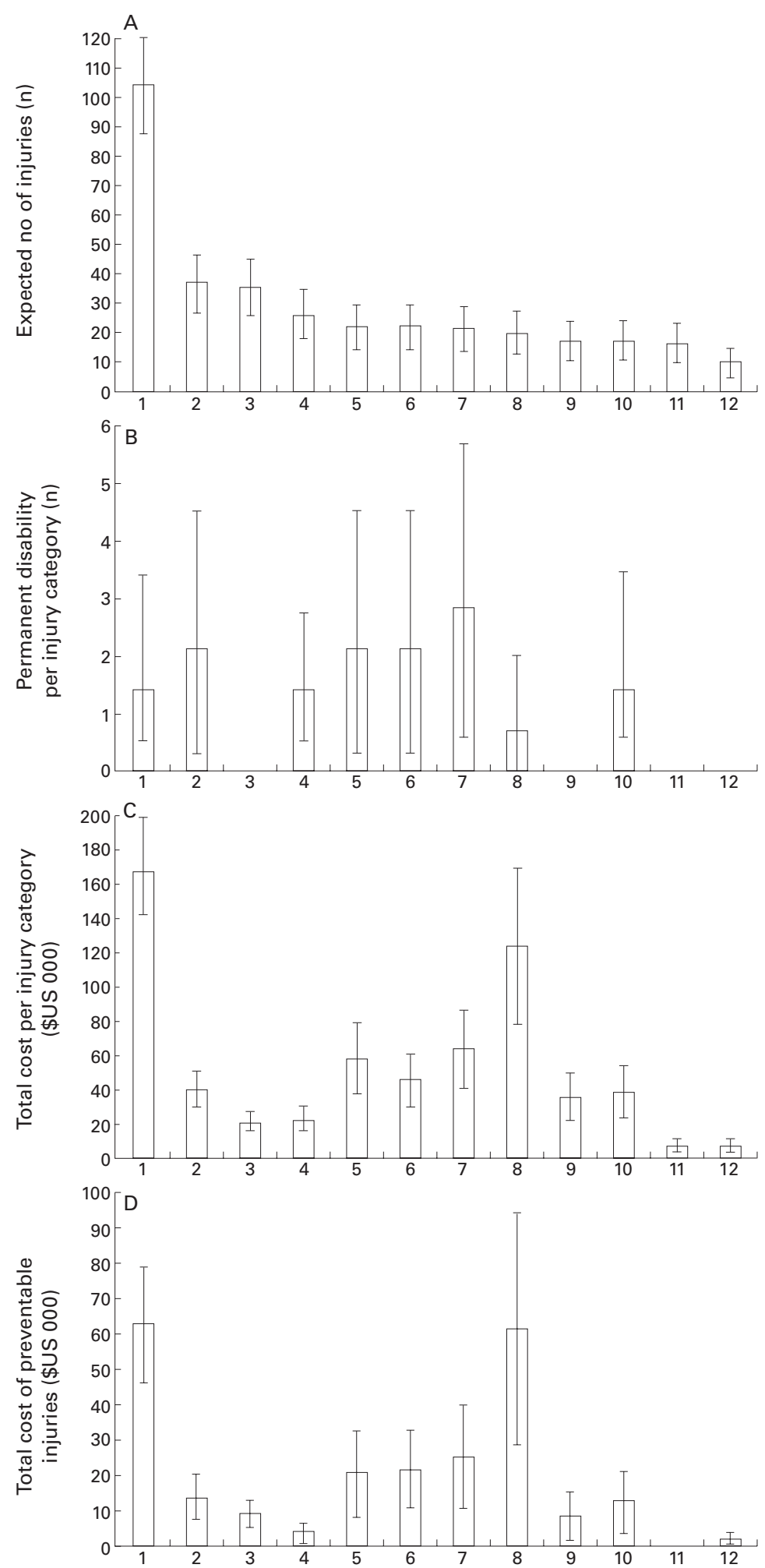

Figure 1 (A) Expected incidence, (B) permanent disability, (C) total average cost per category, and (D) total average cost of preventable injuries per category for every 10000 hospital discharges. 1 = wound infections; $2=$ pressure sores; $3=$ urinary tract infections; $4=$ inadequate manipulation of fractures; $5=$ pulmonary embolism; $6=$ unnecessary operations; $7=$ falls admitted; $8=$ warfarin related; $9=$ bleeding due to non-steroidal anti-inflammatory drugs; $10=$ deep vein thrombosis; $11=$ postoperative nausea and vomiting; 12 = pneumothorax.

data were further adjusted to reflect age, sex, and related co-morbidity. The cost of preventable injuries provides an indirect measure for potential cost savings.

\section{Results}

Figure 1 presents the expected incidence, permanent disability, total average cost per category, and total average cost for preventable injuries within each category per 10000 hospital discharges. Costs are in US dollars (1998) and were converted using an exchange rate of $\$ A U S 1.00=\$ U S 0.645$ (average for 1998). Alternatively, the reader can use purchasing price parities (PPP) for health services of \$AUS $1.00=\$$ US $1.36 .^{15}$

Wound infections were the most common with an incidence of about 100 per 10000 hospital discharges compared with 10-40 per 10000 for the other categories. The associated permanent disability was also significant with an incidence of 1-3 per 10000 hospital discharges. Information on total average cost (fig 1C) and cost of preventable injuries (fig 1D) shows a different profile. Falls were associated with the highest incidence of disability (fig 1B) and ranked high for costs and preventability, despite a lower incidence. Similarly, warfarin related injuries had a high total cost and high cost of preventability despite a relatively low incidence and associated disability.

The total average costs for treating these 12 conditions is estimated at \$US 636000 per 10000 hospital discharges. The cost of the avoidable injuries is over a quarter of a million dollars, equivalent to $2-3 \%$ of the annual budget for a typical Australian community hospital with 120 beds.

\section{Discussion}

The cost of injuries imposes a significant economic burden on both hospitals and the community. This additional information can assist health care policy makers, hospital administrators, and clinicians in setting priorities for programmes that can minimise their impact. While the costs of treatment should not replace incidence data, morbidity data, or quality of life considerations in making choices and decisions about research or prevention priorities, they should not be ignored. This is especially the case where the relative rankings of epidemiology and costing data vary. ${ }^{16}{ }^{17}$ For example, falls, unnecessary operations, and warfarin related injuries are less common events but have significant disability and costs. Rather than directing scarce resources available for prevention programmes towards categories based upon incidence or disability rates alone, these resources could be more efficiently used by targeting highly preventable and costly injuries.

While it is highly desirable to minimise the extent of iatrogenic injuries, they vary in their level of avoidability. Furthermore, there will be a cost associated with programmes that are implemented to reduce their incidence. These issues will also need to be considered in determining priorities for action.

HOW WILL THE COSTING INFORMATION BE INTERPRETED?

Knowing the cost of treating different categories of injuries allows decision makers such as hospital administrators to make more informed 
choices about which injuries are worth targeting for prevention. From the perspective of both the hospital and health funders, information on the costs of treating each category of iatrogenic injuries can help promote technical efficiency or determine how to maximise the preferred outcome-for instance, eliminating preventable injuries or treating nonpreventable injuries - at minimum cost. From the perspective of a health care system, patients and society in general, the preferred outcome would be one aimed at maximising human health and welfare. ${ }^{18}$

Hospitals will be influenced by how the costs of prevention programmes aimed at reducing injuries compare with the cost of treating these injuries. They may want some estimate of cost savings and advice as to which programmes should be implemented, expanded, or contracted and by how much. The cost of strategies or interventions to reduce an injury category will be weighed up against the direct cost savings from averting the injury.

Health insurers and government departments of health will be interested in both immediate direct costs of acute care and the ongoing community and allied health costs of the injuries. This study provides no estimate of the latter costs but includes general practitioner visits, pharmaceuticals, outpatient appointments, physiotherapy, and domiciliary care. The view of a social policy maker may well be "should resources be devoted to minimising iatrogenic injuries as opposed to other areas of health care?" Inclusion of all costs from the perspective of society in general (including indirect and intangible costs) will help policy makers to make resource allocation decisions (allocation efficiency) as to whether

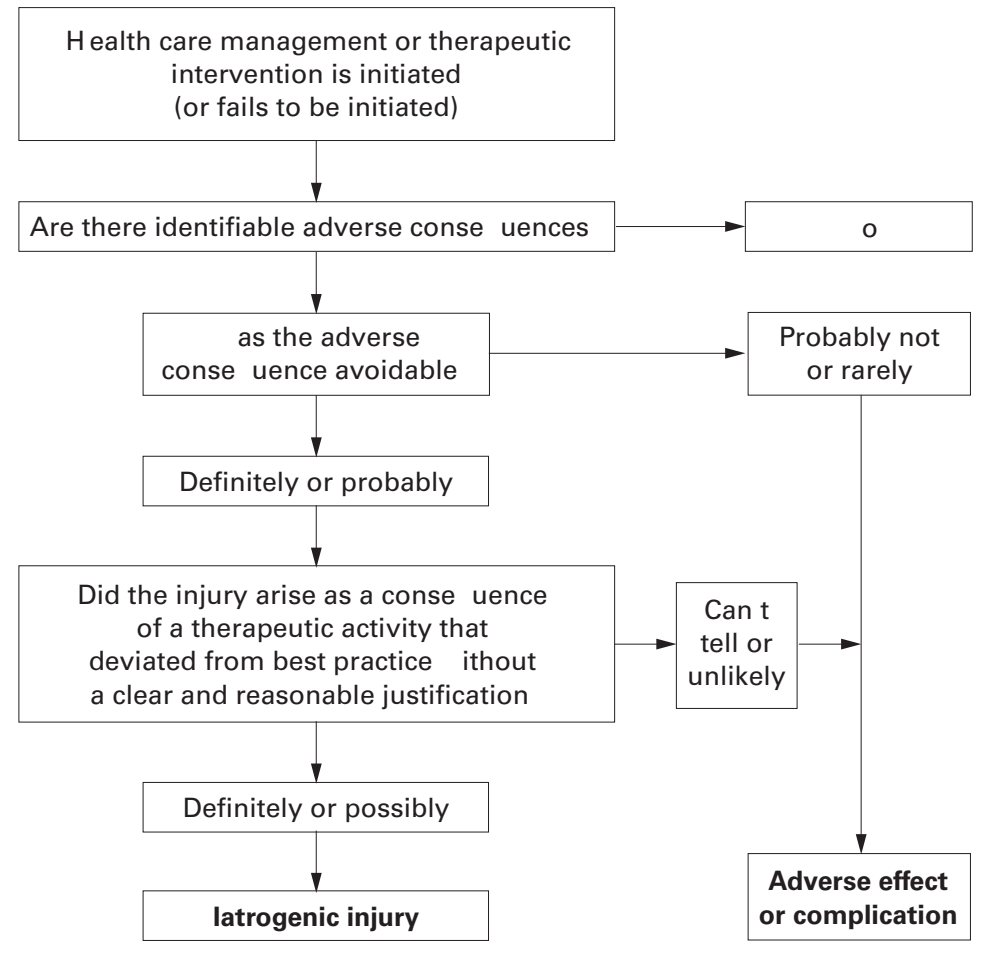

Figure 2 Determination of iatrogenic injuries. or not resources should be devoted to ameliorating them.

The decision on which costs to include and how to value and measure them from an agency perspective such as a hospital or general practice is fairly straightforward and noncontroversial. This is not the case when costing these injuries from a societal perspective. This analysis requires information on the costs that fall not only on the health care sector, but also on health care workers, families, patients (travel and waiting times, pain, suffering, loss of income), and other sectors such as the costs of litigation and lost productivity for employers. Intangible and indirect costs may well be substantial. For example, lifetime costs of road accident victims in Australia and drug related injuries in the USA cost \$US 3.7 billion per annum and US $\$ 2.0$ billion per annum in lifetime costs, respectively. ${ }^{19} 20$

There will be important methodological issues to address when considering the full costs to society. These relate to the dangers of double counting and not distinguishing between resource costs and transfer payments. Whereas transfer payments include monetary compensation, resource costs are those that relate to the process of litigation (court and lawyer costs). Double counting is likely to become a more complicated issue, for example, when outcome measures such as quality of life years (QALYs) are used in the economic evaluation of prevention or risk management programmes.

\section{LIMITATIONS}

Our study has a number of limitations. Accurate costing information is dependent upon good quality epidemiological data. There may be considerable diversity of opinion about whether an adverse effect constitutes an iatrogenic injury, especially injuries involving a human component. We suggest that it would be useful to separate out the adverse effects that may arise in spite of the diligent application of best practice from the consequences of unintended or inappropriate therapeutic interventions. Three explicit questions should be considered and are outlined in fig 2 .

(1) Is the incident associated with adverse consequences to the patient? While this may appear self-evident, there may be some contention about the existence, nature, and extent of an injury that has been suffered by the patient. This is frequently the substance of many medicolegal debates.

(2) Is the injury avoidable? While all adverse effects arising from therapeutic actions by health care providers may be considered iatrogenic, adverse consequences that cannot be foreseen or avoided are an unfortunate but not infrequent occurrence and are considered by most clinicians when weighing up the costs and benefits associated with different therapeutic interventions. True iatrogenic injuries are those that can be largely avoided.

(3) Did the iatrogenic injury arise as a consequence of therapeutic activity that 
deviated from current best practice (as determined by clinical practice guidelines or expert consensus), without clear and reasonable justification? Adverse effects that arise regardless of the careful application of best practice should be a stimulus to seeking better health care interventions rather than labelled as an iatrogenic injury. While all health care providers should pay heed to "doing no harm", all therapeutic interventions are associated with both positive and negative consequences. The iatrogenic component of an adverse event is the negative consequences which are considered to be avoidable.

Both the Australian (QAHCS) and US (UTCOS) surveys have addressed whether the patient suffered adverse consequences and whether the injury was avoidable. Neither attempted to determine whether the event deviated from best practice that could not be justified. True iatrogenic injuries that arise from poor, inadequate, inappropriate, or unnecessary therapeutic interventions should be separated out from unavoidable adverse effects of complications that occur regardless of the application of effective and appropriate interventions by health care providers.

Another limitation of this study is the accuracy of determining both disability and preventability in the QAHCS. While the method used to determine these categories was both explicit and robust, further representative surveys in different health care settings and countries will help to determine whether the categories are both replicable and valid. Levels of agreement should be explicitly reportedfor example, kappa values or intraclass correlation coefficients - and the nature of any discrepancy fully explored.

A third limitation is the problem associated with the currently available costing information. AN-DRGs do not reflect resource use (or the opportunity cost of health care resources) and only direct acute care costs of managing the injury are included. Despite this shortcoming, AN-DRGs do represent health care expenditure imposed on the health care system and society. From a health system or societal perspective, health and welfare measures such as quality of life or disability adjusted life year outcome measures should be included together with economic and epidemiological data in making priority decisions. ${ }^{18}$

As outlined above, AN-DRGs are based on averages and are generally calculated by the top down method. ${ }^{14}$ Patient specific (bottom up) resource use costing data will be necessary if policy and decision makers want to ensure that injuries are minimised or eliminated in an efficient manner. Accurate bottom up costs are particularly pertinent where injuries are not preventable as the treatment of injuries may be managed with greater cost effectiveness by measuring and evaluating each cost component involved in their treatment. For example, the major costs involved in the treatment of wound infections are intravenous antibiotics and hospital bed days. ${ }^{21}$ Switching to oral antibiotics and home care, where appropriate, has the potential to reduce significantly the cost of treating unavoidable wound infections.

\section{Conclusion}

The importance of costing iatrogenic injuries cannot be underestimated. They not only cause considerable morbidity and mortality, but also impose a significant economic burden on both society and the individual (patient, family, health care workers) in terms of consumption of health care resources and lost productivity, and in many cases avoidable pain and suffering. There is a strong case to consider the cost of injuries in future economic evaluations. For example, preliminary estimates of the incidence of warfarin related injuries indicate that they can be up to $7-8 \%$ higher than reported adverse or side effects currently used in some health economic evaluations. ${ }^{22-25}$

AN-DRGs are readily available and easy to calculate and provide an efficient method of quickly detecting areas for research and target setting. However, patient specific costings may be necessary when weighing up the costs and benefits of prevention programmes and when estimating the overall costs to society.

An ongoing national database of iatrogenic injuries is necessary to assist in identifying injuries, to monitor trends, and to provide data for estimating their incidence and associated costs. Such a database is also essential for determining the incidence of injuries related to current therapeutic interventions and newly introduced technologies, pharmaceuticals, or practices where a fragmented approach is unlikely to identify these injuries in a timely fashion or to provide feedback quickly enough to avert further injuries. However, any survey of iatrogenic injuries should define carefully and explicitly what constitutes an injury.

For policy makers, government, decision makers, patients, and their families the most important outcome is the minimisation or, ideally, elimination of these injuries.

1 Wilson RM, Runciman WB, Gibberd RW, et al. The Quality in Australian Health Care Study. Med $\mathcal{f}$ Aust 1995;163:458-71.

2 Leape LL, Brennan TA, Laird N, et al. The nature of adverse events in hospitalised patients: results of the Harvard Medical Practice Study 11. N Engl $\mathcal{f} \mathrm{Med}$ 1991;324:377-84

3 Australian Patient Safety Foundation. Iatrogenic injuries in Australia. Commonwealth Government Report, October 1999.

4 Brennan TA, Leape LL, Laird NM, et al. Incidence of adverse events and negligence in hospitalised patients. $N$ Engl f

5 Wilson RMcL, Harrison BT, Gibberd RW, et al. An analysis of the causes of adverse events from the quality in health of the causes of adverse events from the
care study. Med $\mathcal{F}$ Aust 1999;170:411-5.

6 Drummond MF, O'Brien B, Stoddart GL, et al. Methods for the economic evaluation of health care programmes. 2 nd ed. Oxford: Oxford University Press, 1997. 7Stedman's Medical Dictionary. 26th international ed. Baltimore: Williams and Wilkins, 1995

8 Darchy B, LeMiere E, Figueredo B, et al. Iatrogenic diseases as a reason for admission to the intensive care unit. Arch Intern Med 1999;159:71-8.

9 Bootman LJ, Harrison DL, Cox E. The health care cost of drug-related morbidity and mortality in nursing facilities. Arch Intern Med 1997;157:2089-96.

10 Couch NP, Tilney NL, Rayner AA, et al. The high cost of low-frequency events: the anatomy and economics of surgical mishaps. $N$ Engl f Med 1981;304:634-7.

11 Johnson WG, Brennan TA, Newhouse JP, et al. The economic consequences of medical injuries. Implication for a no-fault insurance plan. $\mathcal{F A M A}$ 1992;267:2487-92. 
12 Leape LL, Lawthers AG, Brennan TA, et al. Preventing medical injury. Qual Rev Bull 1993;May:144-9.

13 Unwin E Codde J. Estimating the cost of hospital treatment for injuries using linked morbidity data. Aust NZ $\mathcal{F}$ Public Health 1998;22:624-6.

14 Commonwealth Department of Health and Family Services. Case mix development program: report on the development of $A N-D R G$ version 3.1 cost weights. Commonwealth Government Publication, August 1998

15 OECD (website: www.oecd.com)

16 Drummond M. Cost-of-illness studies. PharmacoEconomics 1992;2:1-4.

17 Shiell A, Gerard K, Donaldson C. Cost of illness studies: an aid to decision-making? Health Policy 1987;8:317-23.

18 Sheldon T. Promoting health care quality: what role performance indicators? Quality in Health Care 1998; 7(Suppl):S45-50

19 Bates DW, Spell N, Cullen DJ, et al. The costs of adverse events in hospitalised patients. $\mathscr{F} A M A$ 1997;227:307-11.

20 Moller J. Estimating lifetime costs of injury in Australia. Based upon data in "The cost of injury in Victoria", Report
24. Victoria, Australia: Department of Human Services, December 1997.

21 Coello R, Glenister H, Fereres J, et al. The cost infection in surgical patients: a case-control study. $\mathcal{F}$ Hosp Infect 1993;25:239-50.

22 Mol WEM, Egberts TCG. Prophylaxis for venous thromboembolism in hip fracture surgery: total costs and cost effectiveness in The Netherlands. PharmacoEcon 1994;5:48-55.

23 Eckman MH, Falk RH, Pauker SG. Cost-effectiveness of therapies for patients with nonvalvular atrial fibrillation. Arch Intern Med 1998;158:1669-77.

24 Gage BF, Cardinalli AB, Albers GW, et al. Costeffectiveness of warfarin and aspirin for prophylaxis of stroke in patients with nonvalvular atrial fibrillation. $¥ A M A$ 1995;274:1839-45.

25 Gustafsson C, Asplund K, Britton M, et al. Cost effectiveness of primary stroke prevention in atrial fibrillation: Swedish national perspective. BMF 1992;305: 1457-60. 